\title{
EDITORIAL
}

\section{Evidence Based Patient-Centered Care: Are We Ready?}

\section{Ali Baziad}

We already discussed the importance of evidence-based medicine in our previous issue and presumably, we had all agreed to apply it in our daily practice. However, medicine, as we all know, is an ever-changing and ever-evolving subject. As recent conversation arises in the field, it is thought that simply performed medicine based on the most reproducible and factual evidences may not be enough.

Evidence based medicine is a process of turning clinical problems into questions and then systematically locating, appraising, and using related research findings as the basis for clinical decisions. ${ }^{1}$ Meanwhile, patient centered medicine focuses primarily on psychosocial issues, using explicit informed consent, shared decision making and empowering the patient, in order to assure the patients preferences are taken into account. ${ }^{1}$

At first, it is thought that these two methods were two separate things. ${ }^{2}$ One is aimed at the generalization of medicine, and one is aimed into the personalization of medicine. In evidence-based medicine, the randomized controlled trial is thought as the highest level of evidence, while patient based medicine encourages doctor to look at the patient personally, meaning that the best approach is a case by case report. ${ }^{2}$ Fortunately, that debate has been laid to rest; proponents of evidence-based medicine now accept that a good outcome must be defined in terms of what is meaningful and valuable to the individual patient. ${ }^{3}$

In order to make a clinical expertise, there are three things that must be taken into consideration. ${ }^{4}$ First, the patient's clinical circumstance, what is wrong and what treatments options are available. Second, the evidence of the treatment options, how is the efficacy, efficiency and effectiveness of each options. Last, the patient's preference, what the patient could and could not accept. ${ }^{4}$ In short, a doctor must know both the patient and the evidence well to make the best expertise, so the patient could decide what is best for them as a whole person, and not just for the disease. ${ }^{5}$

For this approach to work, it is very important to eliminate the differences between doctors who carry out the research and those who do not. That is one of the objectives of Indonesian Journal of Obstetrics and Gynecology. We try to bridge the gap and share the latest research to every Obstetrics and Gynecology practitioner in Indonesia.

In this issue, we have several interesting scientific researches such as the level of thyroid peroxidase in imminent abortion, the level of total calcium and hypotonic uterus in delivery, and many more. We also have a clinical research article, which analyze the risk and safety in the management of preeclampsia and a literature review on the management of abnormal cytology result.

We would like to express our gratitude to the writer for submitting these articles and sharing their knowledge with all of us. And we would like to ask all the other practitioners, whether you are in the university hospital, private hospital or a private clinic, that we encourage you to submit articles, be it a scientific research, or even an interesting case report. Because as the recent development of patient-centered medicine suggest, even the simplest case report can have a big impact on the management of a patient.

\section{References}

1. Bensing J. Bridging the gap. The separate worlds of evidence-based medicine and patient-centered medicine. Patient Education and Counseling. 2000; 39: 17-25

2. Sacristan A Jose. Patient-centered medicine and patient-oriented research: improving health outcomes for individual patients. BMC Medical Informatics and Decision Making. 2013; 13(6): 2-8

3. Eipsten RM, Street RL. The Values and Value of Patient-Centered Care. Ann Fam Med 2011; 9:100-3.

4. Haynes BR. Physicians' and patients' choices in evidence based practice. BMJ 2002; 324:1350

5. Lacy NL, Becker El. Evidence-based and Patient-centered Care: Results From an STFM Group Project. Fam Med 2008; 40(6): 417-22. 